\title{
Treatment of Anterior Vaginal Wall Prolapse Using Transvaginal Anterior Mesh With Apical Fixation: A Prospective Multicenter Study With up to 2 Years of Follow-up
}

Paulo César Rodrigues Palma ${ }^{1}$, Marilene Vale de Castro Monteiro ${ }^{2}$, Marta Alicia Ledesma ${ }^{3}$, Sebastián Altuna ${ }^{4}$, Juan José Luis Sardi ${ }^{5}$, Cássio Luís Zanettini Riccetto ${ }^{1}$

${ }^{1}$ Universidade Estadual de Campinas (UNICAMP), Campinas, Brazil

${ }^{2}$ Universidade Federal de Minas Gerais (UFMG), Belo Horizonte, Brazil

${ }^{3}$ Clínica Sucre, Córdoba, Argentina

${ }^{4}$ Hospital Universitario Austral, Buenos Aires, Argentina

${ }^{5}$ Hospital Británico, Buenos Aires, Argentina

Purpose: To evaluate the safety and efficacy of a surgical polypropylene mesh for correction of anterior vaginal prolapse, with or without apical defects, by providing simultaneous reinforcement at the anterior and apical aspects of the vagina with a single-incision approach.

Methods: This was a prospective, multicenter, single-arm study involving women with baseline stage $\geq 2$ anterior and/or apical vaginal wall prolapse according to the Pelvic Organ Prolapse Quantification (POP-Q) system. The primary endpoint was defined as achievement of POP-Q stage $\leq 1$ status. Additionally, patient-reported outcomes were assessed using the International Consultation on Incontinence Questionnaire-Vaginal Symptoms (ICIQ-VS). The device under evaluation was Calistar A, which is fixed posteriorly to the sacrospinous ligaments with a novel tissue-anchoring system (TAS) and anteriorly to the obturator internus muscles. Postoperative follow-ups were scheduled at 7 days and at 6, 12, and 24 months.

Results: Ninety-seven women were treated and assessed for the primary outcome. They were followed for up to 2 years ( $n=43)$, with a median of 12 months. Objective cure was achieved in 86 of the 97 patients $(88.7 \%)(\mathrm{P}<0.0005)$. The mean reduction in the ICIQ-VS scores was in the range of $70 \%-90 \%$ for every time point $(\mathrm{P}<0.05)$. No bleeding or surgical revision was reported. Mesh exposure occurred in 7 patients $(7.2 \%)$, urinary retention in $5(5.2 \%)$, de novo dyspareunia in $3(3.1 \%)$, and urinary tract infections in 7 (7.2\%).

Conclusions: This midterm follow-up showed that apical and anterior vaginal reinforcement with a polypropylene implant fixed with a TAS provided good anatomical correction, with no major complications.

Keywords: Cystocele; Pelvic organ prolapse; Surgical mesh; Uterine prolapse; Pelvic floor; Urinary incontinence

- Research Ethics: The study was registered in the Clinical Trial Registry (ICTRP-WHO)/(ICMJE): ACTRN12610000879066. All patients received detailed information about the procedure and provided written informed consent.

- Conflict of Interest: No potential conflict of interest relevant to this article was reported.

Corresponding author: Cassio Luis Zanettini Riccetto

(D) https://orcid.org/0000-0002-2428-3071

Universidade Estadual de Campinas (UNICAMP), Rua R. Vital Brasil, 250,

Campinas SP 13083-590, Brazil

E-mail: cassioriccetto@gmail.com / Tel: +55-35217481 / Fax: +55-35217481

Submitted: February 1, 2018 / Accepted after revision: August 25, 2018 


\section{INTRODUCTION}

Pelvic organ prolapse (POP) is a common condition involving the female pelvic floor and may negatively affect women's quality of life, sexuality, and body image. The prevalence of POP varies depending on the diagnostic criteria, from $3 \%-6 \%$ based on symptoms to $50 \%$ when based upon vaginal examination findings $[1,2]$.

The risk factors for POP vary among patients, and should be evaluated accordingly. A recent systematic review of studies carried out in developed Western countries found that parity, vaginal delivery, age, and body mass index were significant risk factors for primary POP [3]. In addition, fascial repair in traditional operations to correct genital prolapse rely on tissues that are damaged or weakened (due to collagen deficiency, laxity, or poor-quality fascia), and may fail to provide a long-lasting anatomical correction [3], leading to a recurrence rate that can be as high as $40 \%$ [4]. In contrast, the use of mesh with simultaneous surgical correction of apical defects through an abdominal route has been associated with higher anatomical success rates, which has prompted the development of transvaginal techniques using mesh [5]. In 2011, in reply to concerns raised by the U.S. Food and Drug Administration (FDA) [6], the International Urogynecological Association (IUGA) stated that the use of transvaginal mesh is likely to be beneficial in cases involving recurrence of anterior and apical prolapse, a chronic rise in abdominal pressure, scarred or poor-quality native tissue, and/or stage $\geq 2$ anterior prolapse, in which the benefits of using a mesh may outweigh the risks of its main potential complications (dyspareunia, mesh exposure, pelvic pain, and mesh retraction) $[7,8]$. In the last revision of the Cochrane Review (2016), an analysis of 21 randomized clinical trials revealed a lower risk of recurrence of anterior vaginal compartment prolapse when transvaginal mesh was used (11\%-20\%) than when native tissue was used (38\%), with an associated risk ratio of 0.40 (95\% confidence interval, $0.30-0.56$ ) in a sample of 1,494 women [5].

To reduce the incidence of complications, a technique for correction of the apical and anterior compartments was developed using mesh and a single-incision vaginal approach [9]. Damage to adjacent anatomical structures can be reduced by eliminating the need for the blind passage of needles toward the obturator foramen [7]. Using this approach, Moore et al. [10] observed an objective cure in $91.7 \%$ of their patients, insignificant bleeding, and absence of mesh exposure in the vaginal mucosa after a mean follow-up of 13.4 months. Calistar A
(Promedon, Córdoba, Argentina) consists of a macroporous monofilament polypropylene mesh for the correction of anterior and apical vaginal prolapse. It is bilaterally anchored to the sacrospinous ligaments and obturator internus muscles by a novel tissue-anchoring system (TAS) fixation device and multipoint fixation columns, respectively. The aim of the present study was to evaluate the safety and efficacy of this device through objective and subjective patient-reported outcomes. To the authors' knowledge, this is the first report to present an evaluation of this device in a prospective, multicenter study.

\section{MATERIALS AND METHODS}

Five tertiary referral centers participated in this multicenter, singlearm, prospective study. The study was registered in the Clinical Trial Registry (ICTRP-WHO)/(ICMJE): ACTRN12610000879066. All patients received detailed information about the procedure and provided written informed consent.

The inclusion criteria were adult women ( $\geq 40$ years) with stage $\geq 2$ anterior and/or apical vaginal prolapse in accordance to the POP-Quantification (POP-Q) system $(\mathrm{Ba} \geq-1 \mathrm{~cm})[11]$, with no plan for future pregnancy, without comorbidities contraindicating anesthesia, and without diseases involving pelvic tumors. Women were excluded from the study if point $\mathrm{Bp}>-1$ $\mathrm{cm}$ (POP-Q); if simultaneous hysterectomy was indicated at the time of prolapse repair; if they complained of recurrent urinary infections or vulvovaginitis, a compromised immune system, previous radiotherapy, or prolapse recurrence after previous surgical treatment; or if they refused to participate in the study. The primary endpoint was objective cure (anatomical repair) defined as achievement of anterior/apical POP-Q stage $\leq 1$ postoperatively, i.e., point $\mathrm{Ba}<-1 \mathrm{~cm}$ and/or $\mathrm{C}<-1 \mathrm{~cm}$. The International Consultation on Incontinence Questionnaire-Vaginal Symptoms (ICIQ-VS), when available, was also analyzed [12].

The preoperative evaluation involved a urogenital examination, including the POP-Q measurements and a stress test after prolapse reduction for assessing concomitant or occult stress urinary incontinence (SUI). All patients who complained of SUI also answered the International Consultation on Incontinence Questionnaire - Short Form (ICIQ-SF) [13]. Postoperative follow-up examinations were scheduled at 7 days and at 6 , 12 , and 24 months.

The prosthesis under evaluation was Calistar A, a single-incision repair system (Fig. 1) that consists of a type I polypropylene mesh containing 2 polypropylene auto-fixating arms for at- 

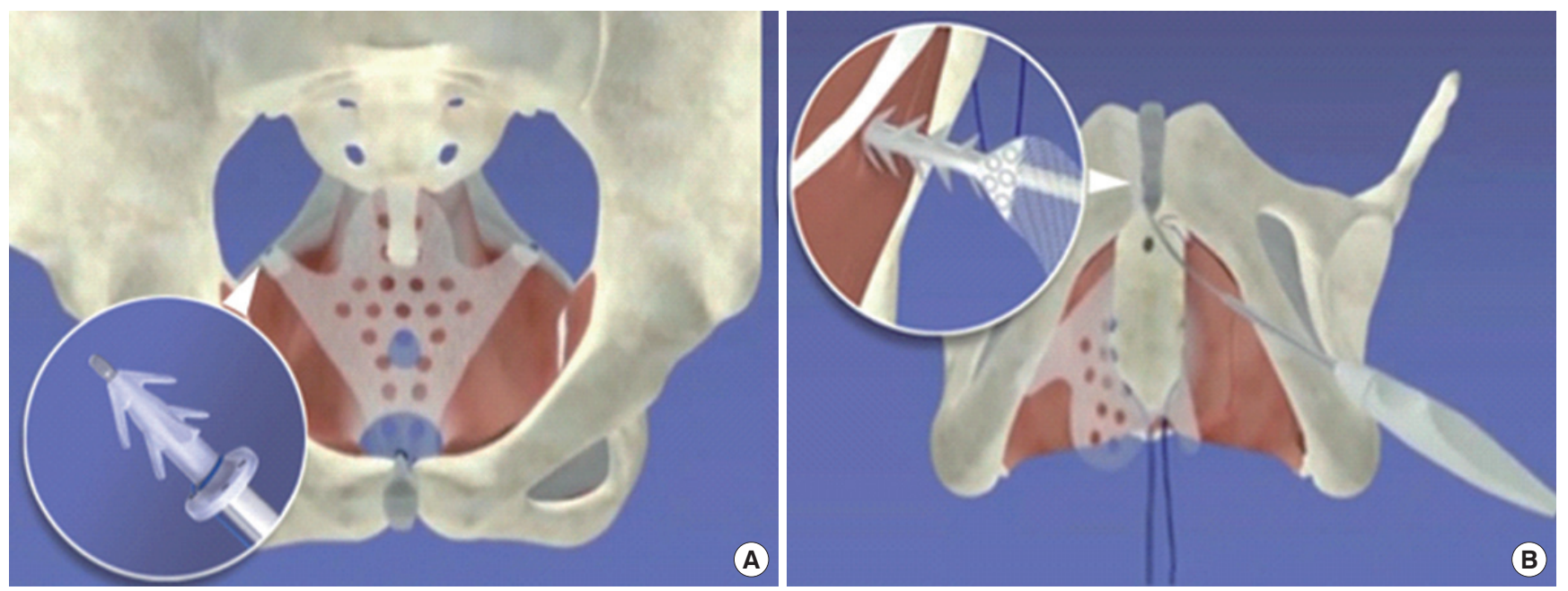

Fig. 1. Calistar A mesh. (A) Tissue anchoring system loaded on the retractable insertion guide for sacrospinous ligament fixation and (B) autofixation device being introduced in the obturator internus muscle.

tachment to the obturator internus muscles, 2 separate polypropylene anchoring devices for fixation onto sacrospinous ligaments, referred to as its TAS, and a retractable insertion guide for placing the anchoring devices. The TAS anchors are specifically designed for fixation at the sacrospinous ligament, with 6 concentrically located spikes arranged at $360^{\circ}$ and a base with a flat rim to limit the depth of insertion into the ligament. The TAS anchors are attached to a 0-0 polypropylene thread that is subsequently used for fixation of the posterior arms of the implants.

\section{Operative Technique}

The patients underwent general anesthesia and were placed in the lithotomy position. A Foley catheter was inserted and left in place. Hydrodissection of the anterior vaginal wall with physiological saline solution was performed. A longitudinal incision was performed from the midurethra to the uterine cervix or vaginal apex with bilateral dissection. Using the index finger, the surgeon performed blunt dissection at the medial edge of the ischiopubic rami, progressing toward the ischial spines until identifying the sacrospinous ligaments bilaterally. The dissection was extended to the uterine cervix or vaginal vault scar caused by a previous hysterectomy and continued until exposure of the pericervical ring.

To implant the prosthesis, the TAS first had to be loaded on the retractable tip of the insertion guide, and then the instrument was guided towards the ligament. Once the TAS was introduced into the ligament, the tip was retracted by a sliding knob on the handle to release the TAS. The procedure contin- ued with the anterior fixation of the mesh, achieved by placing the auto-fixating arms bilaterally into the obturator internus muscles, using the same retractable insertion guide. Two stiches were made with absorbable sutures on both sides of the midurethra to secure the implant and to avoid unwanted displacement. Then, the polypropylene threads of the TAS anchors fixed at the sacrospinous ligaments were used to simultaneously reduce the anterior and apical prolapses to the desired position. Subsequently, the posterior portion of the mesh was sutured with 3-0 polypropylene thread in the pericervical ring or hysterectomy scar.

The vaginal incision was sutured using 2-0 absorbable thread (Caprofyl, Ethicon Inc., subsidiary of Johnson \& Johnson, New Brunswick, NJ, USA). Cystoscopy was left to the surgeons' discretion. The Foley catheter was left in place for 24 hours, and the postvoid residual urine volume was then measured. Patients were informed about the possibility of urinary incontinence persistence postoperatively, in which case they would receive anti-incontinence treatment.

Concomitant posterior prolapse, when present and clinically relevant, was simultaneously corrected through a site-specific defect approach.

\section{Statistical analysis}

No sample calculation was performed and the patients' enrollment was defined based on a time period of 12 months. The statistical analysis was performed with IBM SPSS Statistics ver. 22.0 (IBM Co., Armonk, NY, USA). For longitudinal comparisons, 
the paired Student $t$-test or the Wilcoxon nonparametric test was used for continuous variables, and the McNemar test was used for categorical data. Normality of the distribution of continuous data was assessed with the Shapiro-Wilk test. All pairwise comparisons between more than 2 groups were adjusted using the Bonferroni correction. Multivariable logistic regression analyses were performed to evaluate potential associations among baseline variables and either the objective (POP-Q) or the subjective (ICIQ-VS) outcomes registered at the 24-month follow-up. These analyses included a first stage in which simple associations were studied (contingency tables and 2-sided Pearson chi-square or Fisher exact test for expected frequencies less than 5) to identify one-to-one relationships among the baseline independent variables and the outcome variables. The signifi-

Table 1. Demographic data of the 101 enrolled patients

\begin{tabular}{lc}
\hline Variable & Value \\
\hline Age $(\mathrm{yr})$ & $61.2 \pm 8.3$ \\
Menopause & $87(86.1)$ \\
Body mass index $\left(\mathrm{kg} / \mathrm{m}^{2}\right)$ & $27.0 \pm 4.3$ \\
Pregnancy & $3(2-5)$ \\
Vaginal delivery & $326(82.3)$ \\
Caesarean & $31(7.9)$ \\
Abortion & $39(9.8)$ \\
Patients with previous surgery $\left.{ }^{\mathrm{a}}\right)$ & $44(43.6)$ \\
Anti-incontinence & $10(9.9)$ \\
Hysterectomy & $22(21.8)$ \\
Others ${ }^{\mathrm{b})}$ & $22(21.8)$ \\
Voiding diary & \\
Pain & $24(23.8)$ \\
Dyspareunia & $23(22.8)$ \\
Dysuria & $12(11.9)$ \\
Nocturia & $13(12.9)$ \\
No. of daytime urinations & $7.4 \pm 2.9$ \\
Losses/day & $0(0-2)$ \\
\hline
\end{tabular}

Values are presented as mean \pm standard deviation, number (\%), or median (interquartile range).

${ }^{\text {a) }}$ Some patients underwent more than one surgery. ${ }^{\text {b) }}$ Other surgeries included those performed in the abdominal, vaginal, or perineal regions. cance level adopted was $0.05(5 \%)$ in all cases.

\section{RESULTS}

A total of 101 patients were recruited for this study from March 2010 to September 2013. The demographic data of these women are shown in Table 1. Four patients were lost to follow-up after the 7-day check-up when they were only evaluated for early postoperative complications. These 4 patients were not considered for the midterm follow-up evaluations. The median follow-up was 12 months (interquartile range, 6-24 months), with 43 women (42.6\%) reaching a follow-up of 24 months.

Based on the last follow-up of each participant, 86 of 97 women (88.7\%) met the cure criterion, while 11 (11.3\%) did not $(\mathrm{P}<0.0005)$. Of those 11 women, $2(2.1 \%$ of the entire sample $)$ showed recurrence of anterior prolapse, and $9(9.3 \%$ of the entire sample) exhibited anatomical improvement (reduction in the POP-Q stage) though they did not meet the cure criterion $(\mathrm{Ba}<-1 \mathrm{~cm})$. If the 4 women who were lost to follow-up were considered as cases of POP recurrence (in an intention-to-treat analysis), then the cure rate would be 86 of 101 (85.1\%) and the noncure rate 15 of 101 (14.9\%). The persistence of the cure rate throughout the follow-up period is presented in Table 2. In addition, the mean values of each measurement point of the POPQ showed significant improvement, with Ba moving up on average from approximately $+3.1 \mathrm{~cm}$ to $-2.4 \mathrm{~cm}$ (Table 3 ). The mean ICIQ-VS scores for each of the follow-up periods showed significant reductions, with minor differences across the postoperative timeframe (Table 4), although the missing data rate reached $22.3 \%$ for patients who completed 24 months of follow-up. The vaginal symptoms and sexual symptoms domains exhibited scores at 12 and 24 months that were slightly lower than the 6-month scores, whereas the quality of life score showed an improvement by the first follow-up that was maintained at the same level throughout the remaining evaluation period.

Baseline variables (age, parity, previous surgical history, and body mass index) were tested to identify possible associations

Table 2. Primary outcome (Pelvic Organ Prolapse Quantification point $\mathrm{Ba}<-1 \mathrm{~cm}$ )

\begin{tabular}{|c|c|c|c|c|c|c|}
\hline \multirow{2}{*}{ Outcome } & \multicolumn{2}{|c|}{6 Months $(\mathrm{n}=83)$} & \multicolumn{2}{|c|}{12 Months $(\mathrm{n}=70)$} & \multicolumn{2}{|c|}{24 Months $(n=43)$} \\
\hline & No. (\%) & P-value ${ }^{\text {a) }}$ & No. $(\%)$ & P-value ${ }^{\text {a) }}$ & No. $(\%)$ & P-value ${ }^{a}$ \\
\hline Cured & $73(88.0)$ & $<0.0005$ & $60(85.7)$ & $<0.0005$ & $37(86.0)$ & $<0.0005$ \\
\hline Not-cured & $10(12.0)$ & & $10(14.3)$ & & $6(14.0)$ & \\
\hline
\end{tabular}

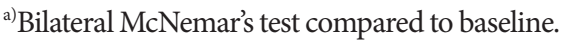


Table 3. POP-Q measurements along the follow-up

\begin{tabular}{|c|c|c|c|c|c|c|c|}
\hline \multirow{2}{*}{ POP-Q } & \multirow{2}{*}{$\begin{array}{c}\text { Preoperative }(\mathrm{n}=97) \\
\text { Mean }(\mathrm{SD})\end{array}$} & \multicolumn{2}{|c|}{6 Months $(\mathrm{n}=83)$} & \multicolumn{2}{|c|}{12 Months $(\mathrm{n}=70)$} & \multicolumn{2}{|c|}{24 Months $(\mathrm{n}=43)$} \\
\hline & & Mean \pm SD & $\mathrm{P}$-value & Mean \pm SD & P-value & Mean \pm SD & $\mathrm{P}$-value \\
\hline $\mathrm{Aa}$ & $1.7(1.2)$ & $-2.0 \pm 0.7$ & $<0.0005$ & $-2.0 \pm 0.8$ & $<0.0005$ & $-2.1 \pm 0.9$ & $<0.0005$ \\
\hline $\mathrm{Ba}$ & $3.1(1.7)$ & $-2.4 \pm 0.8$ & $<0.0005$ & $-2.4 \pm 0.9$ & $<0.0005$ & $-2.3 \pm 1.1$ & $<0.0005$ \\
\hline C & $0.3(3.3)$ & $-6.4 \pm 2.1$ & $<0.0005$ & $-6.2 \pm 2.4$ & $<0.0005$ & $-6.7 \pm 2.4$ & $<0.0005$ \\
\hline Ap & $-0.7(1.6)$ & $-2.2 \pm 0.9$ & $<0.0005$ & $-2.2 \pm 1.0$ & $<0.0005$ & $-2.4 \pm 0.9$ & $<0.0005$ \\
\hline $\mathrm{Bp}$ & $-1.0(2.1)$ & $-2.4 \pm 1.1$ & $<0.0005$ & $-2.4 \pm 1.2$ & $<0.0005$ & $-2.5 \pm 1.0$ & 0.001 \\
\hline $\mathrm{D}$ & $-1.2(5.6)$ & $-7.5 \pm 1.4$ & $<0.0005$ & $-7.5 \pm 1.5$ & $<0.0005$ & $-7.7 \pm 1.8$ & $<0.0005$ \\
\hline gh & $4.2(1.2)$ & $3.1 \pm 0.8$ & $<0.0005$ & $3.1 \pm 0.8$ & $<0.0005$ & $3.4 \pm 0.9$ & $<0.0005$ \\
\hline $\mathrm{pb}$ & $2.8(1.2)$ & $3.2 \pm 0.9$ & 0.002 & $3.2 \pm 0.8$ & 0.004 & $3.4 \pm 0.9$ & 0.087 \\
\hline tvl & $8.4(1.0)$ & $8.2 \pm 1.2$ & 0.072 & $8.1 \pm 1.0$ & 0.019 & $8.3 \pm 1.2$ & 1.000 \\
\hline
\end{tabular}

POP-Q, Pelvic Organ Prolapse Quantification; SD, standard deviation; gh, genital hiatus; pb, perineal body; tvl, total vaginal length.

P-values correspond to a bilateral paired t-test compared to baseline.

Table 4. ICIQ-VS outcome

\begin{tabular}{|c|c|c|c|c|c|c|c|}
\hline \multirow{2}{*}{ ICIQ-VS } & \multirow{2}{*}{$\frac{\text { Preoperative }(\mathrm{n}=64)}{\text { Mean } \pm \text { SD }}$} & \multicolumn{2}{|c|}{6 Months $(\mathrm{n}=49)$} & \multicolumn{2}{|c|}{12 Months $(\mathrm{n}=40)$} & \multicolumn{2}{|c|}{24 Months $(n=33)$} \\
\hline & & Mean \pm SD & P-value ${ }^{a)}$ & Mean \pm SD & $\mathrm{P}$-value ${ }^{\mathrm{a})}$ & Mean \pm SD & P-value ${ }^{a}$ \\
\hline Vaginal symptoms & $25.3 \pm 10.8$ & $8.4 \pm 6.4$ & $<0.0005$ & $4.4 \pm 8.2$ & $<0.0005$ & $5.2 \pm 7.3$ & $<0.0005$ \\
\hline Sexual symptoms & $10.0 \pm 15.3$ & $3.0 \pm 6.5$ & 0.016 & $1.0 \pm 1.3$ & 0.002 & $1.0 \pm 1.3$ & 0.003 \\
\hline Quality of life & $7.6 \pm 6.4$ & $1.1 \pm 2.0$ & $<0.0005$ & $1.2 \pm 2.7$ & $<0.0005$ & $1.5 \pm 2.8$ & $<0.0005$ \\
\hline
\end{tabular}

ICIQ-VS, International Consultation on Incontinence Questionnaire - Vaginal Symptoms; SD, standard deviation.

${ }^{a)}$ Wilcoxon signed ranks test, bilateral. Significance level $\alpha=0.05$.

Table 5. Evolution of patients with concomitant SUI

\begin{tabular}{lcccc}
\hline Variable & Preoperative & 6 Months & 12 months & 24 Months \\
\hline Positive stress test & $43 / 101(42.6)$ & $7 / 33(21.2)$ & $4 / 26(15.4)$ & $4 / 18(22.2)$ \\
P-value & - & $<0.0005$ & $<0.0005$ & $<0.0005$ \\
ICIQ-SF & $13.3 \pm 4.6$ & $2.5 \pm 5.5$ & $2.4 \pm 5.2$ & $3.2 \pm 6.0$ \\
P-value & - & $<0.0005$ & $<0.0005$ & $<0.0005$ \\
\hline
\end{tabular}

Values are presented as number (\%) or mean \pm standard deviation.

SUI, stress urinary incontinence; ICIQ-SF, International Consultation on Incontinence Questionnaire - Short Form.

Comparisons against baseline. Not all women with preoperative SUI completed the subsequent follow-ups, therefore stress test rates were computed with the actual number of patients who complied with the corresponding follow-up.

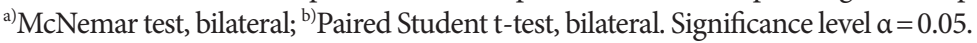

with the outcome variables (POP-Q points $\mathrm{Aa}, \mathrm{Ba}$ and $\mathrm{C}$, and the individual ICIQ-VS scores). Continuous variables were converted to binary variables prior to the tests following clinically relevant criteria (i.e., age: $\geq 60$ or $<60$ years, parity: $\geq 3$ or $<3$ deliveries, previous surgery: yes or no, body mass index: $\geq 30$ or $<30$ $\mathrm{kg} / \mathrm{m}^{2}$, POP-Q Aa, Ba and C $\geq-1 \mathrm{~cm}$ or $<-1 \mathrm{~cm}$, and ICIQ-VS scores: reduction $\geq 50 \%$ or reduction $<50 \%$ ). None of the individual pairs of independent and dependent variables were found to be significantly associated $(\mathrm{P}>0.05)$. Despite failing to find significant associations in any of the simple analyses, multiple logistic regression models were also run with the same independent and dependent variables described earlier for the simple association analyses. None of the regression models conducted for any of these dependent variables yielded significant odds ratios. In other words, no significant associations could be established between the outcome variables and the independent variables. 
Concomitant SUI was confirmed in 43 of the patients (42.6\%). The baseline mean ICIQ-SF score in this subgroup was $13.3 \pm 4.6$. At the last follow-up of each patient, there were 32 negative stress tests $(80.0 \%)$, and 8 positive stress tests (20\%), indicating a significant improvement from baseline $(\mathrm{P}<0.0005)$, and the ICIQ-SF mean score also significantly improved to $3.0 \pm 5.6(\mathrm{P}<0.0005)$. Three of the 43 women with initial SUI were among the 4 patients who were lost to follow-up after the immediate postoperative evaluation. Table 5 summarizes the results of the stress test and ICIQ-SF. SUI treatment was offered to patients who presented a positive stress test after the correction of POP, but only 1 patient received a transobturator sling.

Twelve patients (35.2\%) had preoperative overactive bladder syndrome. Remission of the associated symptoms occurred in 7 patients (58.3\%), and the remaining 5 were treated with anticholinergics. Seven patients (7.2\%) had de novo urgency, and all of them responded to anticholinergics. No visceral injury, bleeding, or need for reoperation due to hematoma formation or pain occurred in any patients. Temporary de novo dyspareunia was reported by 3 women (3.1\%). Urinary tract infections occurred in 7 patients (7.2\%) in the immediate follow-up period and were treated with antibiotics; another 5 patients (5.2\%) presented other infections not related to the surgical site. Five patients (5.2\%) developed postoperative urinary retention. Spontaneous remission occurred in 4 of these 5 patients after 5 days of catheterization. One patient required an incision in the anterior arm of the mesh to resolve the situation. Mesh exposure was observed in 7 patients (7.2\%), requiring resection of the exposed mesh in 5 patients and treatment with topical estrogen in 2 . There was no mesh infection or need for mesh removal in any patients.

\section{DISCUSSION}

The main objective of this prospective study was to characterize the midterm efficacy and safety of Calistar A for the correction of anterior and apical vaginal prolapse. A total of 101 recruited women were initially enrolled, of whom 97 (96.0\%) had postoperative POP-Q assessments. The median follow-up for this cohort was 12 months (interquartile range, 6-24 months), with a subgroup of 43 women (42.6\%) reaching the 24-month follow-up milestone. The primary endpoint was the evaluation of objective cure, defined as POP-Q point $\mathrm{Ba}<-1 \mathrm{~cm}$. Data obtained through the course of the study fulfilled the main objectives, enabling a better understanding of the overall perfor- mance of this synthetic prosthesis.

Objective cure was achieved in 86 patients (88.7\%). These results are comparable to those presented in the 2016 Cochrane Review by Maher et al. [5]. The good and relatively long-lasting anatomical correction was most likely because of the permanent support provided by the polypropylene implant, and the simultaneous correction of the anterior and apical aspects of the vagina. It has been recognized, by imaging techniques [14] and in simulated corrections [15], that adequate vaginal support at level I positively impacts the anterior vaginal wall position. Untreated apical defects may eventually lead to the recurrence of prolapse, even when adequate support is provided to the anterior wall. The TAS and its insertion guide offered an easy and repeatable method for sacrospinous fixation at that level. The absence of intraoperative bleeding or postoperative hematomas needing drainage are thought to be related to the design of the TAS and the insertion guide, which enabled a minimally invasive passage to reach the ligaments of interest. These findings contrast with a reported vascular complication rate of up to $4 \%$ with systems involving double transobturator passage $[10,16]$, and seem to confirm the hypothesis that sacrospinous fixation is safer than the transobturator route [9]. Additionally, there was no need for mesh removal due to pelvic pain, probably due to the absence of nerve injury and mesh retraction, which have been frequently described in studies of the transobturator approach $[17,18]$.

Patient-reported outcomes on the ICIQ-VS significantly improved after the intervention. However, these positive tendencies should be analyzed with caution. The reduced subset of subjects who completed the questionnaire at each follow-up limits the generalizability of these findings.

The device under evaluation has a midurethral portion that can support structures at this level. Though it was not part of the primary objective of this study, the effects of the device on incontinence were evaluated over time. Eighteen of the 43 women with initial SUI completed the 24-month follow-up. Fourteen of those women $(78.8 \%)$ achieved negative stress tests. Similarly, the ICIQ-SF at 24 months was significantly lower than the preoperative value ( 3.2 vs. 13.3). There is at least 1 previous example of treating anterior prolapse and SUI with a polypropylene mesh with retropubic arms that also extended to the middle urethral portion; this technique showed a reduction of urinary incontinence from $29.8 \%$ to $1.9 \%$ [19]. Despite these promising results, stronger evidence is necessary before drawing definitive conclusions on the capability of mesh devices to simultaneously treat 
POP and SUI in accordance with this principle.

Postoperative complications associated with synthetic vaginal meshes have been intensely discussed in recent years. A systematic review (2011) revealed that the mean transvaginal mesh exposure rate was $10.3 \%$ for synthetic grafts [20]; however, great variation across the studies was identified. In the present study, the mesh exposure rate was 7.2\%. It should be noted that this cohort included a large number of postmenopausal women (87 [86.1\%]), a population known to be at high risk of exposure [3]. None of the patients were on estrogen replacement therapy before the beginning of the study. The factors that could have helped keep the exposure rate at these levels were hypothesized to be twofold. First, all surgeons had extensive experience in the use of this type of prosthesis before the onset of the study. Secondly, the eligibility criteria may have excluded some populations associated with higher risks of mesh exposure or for whom the benefit/risk ratio of undergoing such a procedure would not have been adequate. The combination of a carefully selected patient and an experienced surgeon are the key factors for ensuring a balanced benefit/risk ratio, as has been recommended after the emergence of widespread concerns about mesh use in POP repair [7]. Another complication worth mentioning is dyspareunia, which appeared de novo in 3 patients $(3.1 \%)$ in this cohort. This low prevalence could be related to the absence of both mesh retraction and general pelvic pain. It also could have been underreported by this population, as a majority of aged postmenopausal women may have found this symptom not to be a burden or may not have felt comfortable reporting it.

The strengths of this investigation include its prospective and multicenter design, which increases its generalizability, although its noncomparative, single-arm approach and the lack of power calculation for sample size determination pose a significant limitation. This investigation was planned prior to the establishment of guidelines by the FDA and IUGA in response to concerns about using mesh prostheses; therefore, its primary objective was mainly focused on anatomical criteria. Moreover, patient-reported outcomes could only be collected from a subset of the original cohort, reducing the possibility for conclusive conclusions to be drawn regarding this issue. The number of patients at each follow-up was not consistent, and the reasons behind this fluctuating compliance must be assessed in the context of particular socioeconomic conditions, distance to the investigational sites, and availability of transportation for certain communities that were involved in this study.
Despite the weakness of the analysis due to the high dropout rate, the results from the participants who could be analyzed indicate that the device under evaluation provided good and lasting anatomical support to correct anterior wall prolapse with or without apical defects, with no major complications for a median period of 12 months postoperatively, and presumably up to 24 months. Further research could add valuable supplemental information to enhance the generalizability of these findings, ideally in the form of a randomized controlled trial with compound endpoints that put a greater emphasis on patient-reported outcomes.

\section{AUTHOR CONTRIBUTION STATEMENT}

- Full access to all the data in the study and takes responsibility for the integrity of the data and the accuracy of the data analysis: PCRP

- Study concept and design: PCRP

- Acquisition of data: PCRP, CLZR, SA, MAL, JJLS, MVCM

- Analysis and interpretation of data: PCRP, CLZR

- Drafting of the manuscript: CLZR

- Critical revision of the manuscript for important intellectual content: PCRP, CLZR

- Statistical analysis: CLZR

- Obtained funding: PCRP, CLZR, SA, MAL, JJLS, MVCM

- Administrative, technical, or material support: CLZR

- Study supervision: PCRP, CLZR

\section{REFERENCES}

1. Barber MD, Maher C. Epidemiology and outcome assessment of pelvic organ prolapse. Int Urogynecol J 2013;24:1783-90.

2. Gyhagen M, Bullarbo M, Nielsen TF, Milsom I. Prevalence and risk factors for pelvic organ prolapse 20 years after childbirth: a national cohort study in singleton primiparae after vaginal or caesarean delivery. BJOG 2013;120:152-60.

3. Vergeldt TF, Weemhoff M, IntHout J, Kluivers KB. Risk factors for pelvic organ prolapse and its recurrence: a systematic review. Int Urogynecol J 2015;26:1559-73.

4. Weber AM, Walters MD, Piedmonte MR, Ballard LA. Anterior colporrhaphy: a randomized trial of three surgical techniques. Am J Obstet Gynecol 2001;185:1299-304.

5. Maher C, Feiner B, Baessler K, Christmann-Schmid C, Haya N, Marjoribanks J. Transvaginal mesh or grafts compared with native tissue repair for vaginal prolapse. Cochrane Database Syst Rev 
2016;2:CD012079.

6. U.S. Food and Drug Administration. Safety communications - UPDATE on serious complications associated with transvaginal placement of surgical mesh for pelvic organ prolapse. Silver Spring (MD): U.S. Food and Drug Administration; 2011.

7. Davila GW, Baessler K, Cosson M, Cardozo L. Selection of patients in whom vaginal graft use may be appropriate. Consensus of the 2nd IUGA Grafts Roundtable: optimizing safety and appropriateness of graft use in transvaginal pelvic reconstructive surgery. Int Urogynecol J 2012;23 Suppl 1:S7-14.

8. Haylen BT, Sand PK, Swift SE, Maher C, Moran PA, Freeman RM. Transvaginal placement of surgical mesh for pelvic organ prolapse: more FDA concerns--positive reactions are possible. Int Urogynecol J 2012;23:11-3.

9. Moore RD, Mitchell GK, Miklos JR. Single-incision vaginal approach to treat cystocele and vault prolapse with an anterior wall mesh anchored apically to the sacrospinous ligaments. Int Urogynecol J 2012;23:85-91.

10. Moore RD, Beyer RD, Jacoby K, Freedman SJ, McCammon KA, Gambla MT. Prospective multicenter trial assessing type I, polypropylene mesh placed via transobturator route for the treatment of anterior vaginal prolapse with 2-year follow-up. Int Urogynecol J 2010;21:545-52.

11. Haylen BT, Maher CF, Barber MD, Camargo S, Dandolu V, Digesu A, et al. An International Urogynecological Association (IUGA)/ International Continence Society (ICS) joint report on the terminology for female pelvic organ prolapse (POP). Int Urogynecol J 2016;27:655-84.

12. Tamanini JT, Almeida FG, Girotti ME, Riccetto CL, Palma PC, Rios LA. The Portuguese validation of the International Consultation on Incontinence Questionnaire-Vaginal Symptoms (ICIQ-VS) for Brazilian women with pelvic organ prolapse. Int Urogynecol J Pelvic Floor Dysfunct 2008;19:1385-91.

13. Tamanini JT, Dambros M, D’Ancona CA, Palma PC, Rodrigues Netto N Jr. Validation of the "International Consultation on Incontinence Questionnaire -- Short Form” (ICIQ-SF) for Portuguese. Rev Saude Publica 2004;38:438-44.

14. Summers A, Winkel LA, Hussain HK, DeLancey JO. The relationship between anterior and apical compartment support. Am J Obstet Gynecol 2006;194:1438-43.

15. Lowder JL, Park AJ, Ellison R, Ghetti C, Moalli P, Zyczynski H, et al. The role of apical vaginal support in the appearance of anterior and posterior vaginal prolapse. Obstet Gynecol 2008;111:152-7.

16. Caquant F, Collinet P, Debodinance P, Berrocal J, Garbin O, Rosenthal C, et al. Safety of Trans Vaginal Mesh procedure: retrospective study of 684 patients. J Obstet Gynaecol Res 2008;34:449-56.

17. Feiner B, Maher C. Vaginal mesh contraction: definition, clinical presentation, and management. Obstet Gynecol 2010;115(2 Pt 1): 325-30.

18. Margulies RU, Lewicky-Gaupp C, Fenner DE, McGuire EJ, Clemens JQ, Delancey JO. Complications requiring reoperation following vaginal mesh kit procedures for prolapse. Am J Obstet Gynecol 2008;199:678.

19. Palma P, Riccetto C, Prudente A, Dalphorno F, Delroy C, Castro R, et al. Monoprosthesis for anterior vaginal prolapse and stress urinary ncontinence: midterm results of an international multicentre prospective study. Int rogynecol J. 2011;22:1535-41.

20. Abed H, Rahn DD, Lowenstein L, Balk EM, Clemons JL, Rogers $\mathrm{RG}$, et al. Incidence and management of graft erosion, wound granulation, and dyspareunia following vaginal prolapse repair with graft materials: a systematic review. Int Urogynecol J 2011;22: 789-98. 